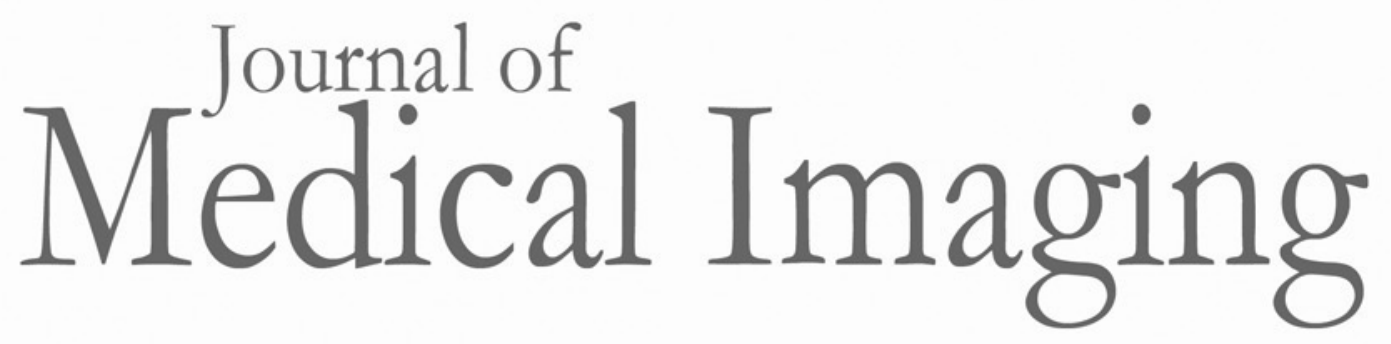

Medicallmaging.SPIEDigitalLibrary.org

\title{
JMI 2016 List of Reviewers
}


The Journal of Medical Imaging would like to sincerely thank the following individuals who served as reviewers in 2016 . The success of our publication hinges on the voluntary contributions of time and energy put forth by these professionals.

\begin{tabular}{|c|c|c|}
\hline Shannon Agner & Guy Cloutier & Xin $\mathrm{He}$ \\
\hline Shaheen Ahmed & Elodia Cole & Tobias Heimann \\
\hline Osman Ahsen & David Cory & Rebekka Heitmar \\
\hline Selim Aksoy & Ian Cunningham & Karl Helmer \\
\hline Bashir Al-Diri & Sonia Dahdouh & Irene Hernandez-Giron \\
\hline Mark Anastasio & Xianjin Dai & William E. Higgins \\
\hline Sameer Antani & Mini Das & Melissa Hill \\
\hline Bhavna Antony & Behdad Dashtbozorg & David Hintenlang \\
\hline Tomoki Arichi & Marleen de Bruijne & Yasushi Hirano \\
\hline Samuel Armato & Tharindu De Silva & Christoph Hoeschen \\
\hline William Auffermann & Luis de Sisternes & Kenneth Hoffmann \\
\hline Mohammad Avanaki & P. Dendooven & Vanessa Holanda \\
\hline Suyash Awate & Henri Der Sarkissian & David W. Holdsworth \\
\hline Ehsan Azimi & Niti Dhutia & Katharina Holland \\
\hline Mahdi Azizian & Robert Dickinson & Mike House \\
\hline George Azzopardia & Xiaowei Ding & Edward Hsu \\
\hline Aldo Badano & Ayush Dogra & Houchun $\mathrm{Hu}$ \\
\hline Cristian Badea & Robert Doot & Chao Huang \\
\hline Jongduk Baek & Karen Drukker & Xiaojie Huang \\
\hline Predrag Bakic & Can Duan & David Allan Hughes \\
\hline Harrison Barrett & David Duncan & Irshad Humayun \\
\hline Bruno Barufaldi & Jan Egger & Q "Ben" Huynh \\
\hline Clement Baumgarten & Issam El Naqa & Humayun Irshad \\
\hline Luc Beaulieu & Rebecca Fahrig & Yuji Iwadate \\
\hline Gert Behiels & Xiaobing Fan & Matthew Jacobson \\
\hline Akram Belghtih & Allan Farman & Jayender Jagadeesan \\
\hline Moshe Ben-David & Jessica Faruque & Babak Jahromi \\
\hline Ewert Bengtsson & Azadeh Firouzian & Andras Jakab \\
\hline M'hamed Bentourkia & James Ford & Andrew Janowczyk \\
\hline Jatta Berberat & Jean-Phlippe Fortin & Abhinav Jha \\
\hline Corinne Berclaz & Eric Frey & Rui Jia \\
\hline Yusuf Bhagat & Clifton David Fuller & Yuhao Jiang \\
\hline Francois Bochud & Maria Gabrani & Jing Jin \\
\hline Sandeep Bodduluri & Brandon Gallas & Yan Jin \\
\hline John Boone & Ziba Gandomkar & Robert Jones \\
\hline Jovan Brankov & Grace Gang & M. Zahangir Kabir \\
\hline Ulf-Dietrich Braumann & Mingchen Gao & Vipin Kamble \\
\hline Alberto Bravin & Bilwaj Gaonkar & Manjunath Kanabagatte \\
\hline Gianni Bussolati & Aimilia Gastounioti & Cheenu Kappadath \\
\hline Brett Byram & Marios Gavrielides & Dimitrios Kapsokalyvas \\
\hline Darrin Byrd & Bruce Gaynes & Andrew Karellas \\
\hline Mariano Cabezas & Jiajia Ge & Karim Karim \\
\hline Jochen Cammin & Arkadiusz Gertych & Joel Karp \\
\hline Guohua Cao & Olivier Gevaert & Sokratis Katsikas \\
\hline Timothy Carroll & Michael Ghaly & Jason Kaufman \\
\hline Ann-Katherine Carton & Howard Gifford & Keigo Kawaji \\
\hline Amine Chaabouni & Fantin Girard & Brad Keller \\
\hline Heang-Ping Chan & Randolph Glickman & Shadab Khan \\
\hline Chien-Ping Chang & Beth Goins & Ali Khazaee \\
\hline Chen Chen & Christian Graff & Takamaro Kikkawa \\
\hline Elvis Chen & Enrico Grisan & Jessica Kishimoto \\
\hline Jingyun Chen & Huifeng Guan & Takayuki Kitasaka \\
\hline Xin Chen & Hua Guo & Janne Koljonen \\
\hline Xinjian Chen & Lubomir Hadjiiski & Sergey Komarov \\
\hline Teodora Chitiboi & Susan Hagness & Despina Kontos \\
\hline Seungryong Cho & Yuji Hatanaka & Ender Konukoglu \\
\hline Matthew Clarkson & Mathieu Hatt & Thijs Kooi \\
\hline
\end{tabular}


Aikaterini Kotrotsou Srilalan Krishnamoorthy Ganapathy Krishnamurthi Elizabeth Krupinski Wattanapong Kurdthongmee David Kwartowitz Manu Lakshmanan Thomas Langerak Maarten Lansberg Guenter Lauritsch Roger Lecomte George Lee Jae Sung Lee Kisung Lee Minho Lee Ting-Yim Lee Yongsook Lee Eelco Lens Nikolas Lessmann Mikolaj Leszczuk Oliver Lezoray

Chiye Li

Hangfan Li

$\mathrm{Li} \mathrm{Li}$

Liang Li

Limin Li

Qin Li

Geert Litjens

Jiamin Liu

Jiangkun Liu

Jun Liu

QiaoHong Liu

Shi Liu

Shuang Liu

Zheng Liu

Joseph Lo

Rodney Long

Cheng Lu

Caroline Magnain

Dwarikanath Mahapatra

Amir Pasha Mahmoudzadeh Stanislaw Majewski James Malcolm Amir Manbachi Luca Marmugi Johanne Martel-Pelletier Robert Marti Marcos Martin-Fernandez John Mashford

Francesc Massanes

Yoshitaka Masutani

Sarah Mattonen

Carlos Mello

Thomy Mertzanidou

Scott Metzler

Michael Miga

Charles Mistretta

Sunanda Mitra

Robert Miyaoka

Decebal Constantin Mocanu
Dimple Modgil

Mehdi Moghari

Jan Moltz

James Monaco

Ramin Moshavegh

Claudia Mota

Henning Muller

Ana Namburete

Rohit Nayak

Hamid Neshat

Muhammad Khalid Khan Niazi

Johan Nuyts

Alessandro Olivo

Daniel Orringer

Xiaochuan Pan

Kerstin Pannek

Felipe Parages

Kedar Patwardhan

Wei Peng

Yahui Peng

Irene Perali

Amy Perkins

Franjo Pernus

Antonios Perperidis

Nicholas Petrick

Aria Pezeshk

Axel Pinz

Ginger Pocock

Killian Pohl

Antonio Porras

Jinyi Qi

Xulei Qin

Arvind Rao

Ingrid Reiser

Lassi Rieppo

Ivan Rosado-Mendez

Georg Rose

Laurence Rouet

Luigi Rovati

Mirabela Rusu

Ken Sakaie

Septimiu Salcudean

Ivan Salgo

Veit Sandfort

Peter Santi

Pinaki Sarder

Saradwata Sarkar

Steven Schalekamp

Helmut Schlattl

Jeffrey Schmall

Taly Schmidt

Ingrid Scholl

Hauke Schramm

loannis Sechopoulos

Baha Sen

Caglar Senaras

Reza Shah-Hosseini

Samir Sharma

Shiwen Shen

John A. Shepherd
Adam Sibley

Emil Sidky

Arkadiusz Sitek

Piotr Slomka

Daniel Smith

U Snekhalatha

Justin Solomon

Aristeidis Sotiras

Rachel Sparks

Stefanie Speidel

Chukka Srinivas

Roman Starosolski

Danail Stoyanov

Nicola Strisciuglio

Nagesh Subbanna

Suleman Surti

Linas Svilainis

Katsuyuki Taguchi

Nima Tajbakhsh

Ashish Tamhane

Likun Tan

Jianbo Tang

Xiangyang Tang

Hideaki Tashima

Sian Taylor-Phillips

Daniel Thedens

John Thompson

Zhiqiang Tian

John Tomaszewski

Turid Torheim

Darren Treanor

Trong-Kha Truong

Timothy Tuinstra

Nicholas Tustison

Giovanni Ughi

James Uhlir

Eranga Ukwatta

Radhika Vadhi

Jeroen van der Laak

Bram van Ginneken

Marijn van Stralen

Harini Veeraraghavan

Prashant Verma

Marcelo Vieira

Satish Viswanath

Irina Voiculescu

Tao Wan

Bo Wang

Guobao Wang

Haibo Wang

Hao Wang

Hongzhi Wang

Li Wang

Zhao Wang

Aaron Ward

Mihir Wechalekar

E. Welch

Daniel Welfer

Roshan Welikala

Kevin Wells 
Gezheng Wen

Bruce Whiting

Mark Williams

Axel Wismueller

Dufan Wu

Jia $\mathrm{Wu}$

Jing Wu

Meng Wu

Shandong $\mathrm{Wu}$

Lei Xi

Guanglei Xiong

Jun Xu

Qiaofeng Xu

Sheng $\mathrm{Xu}$

Dong Yang

Fan Yang
Hao Yang

Li Yang

Zhen Yang

Jianhua Yao

Xincheng Yao

Bulent Yener

Jung Yeol Yeom

Hong-Jun Yoon

Stefano Young

Lifeng Yu

Zhicong $\mathrm{Yu}$

Jing Yuan

Yading Yuan

Stefan Zachow

Constantine Zakkaroff

Wojciech Zbijewski
Rongping Zeng

Bailing Zhang

Hanming Zhang

Jing Zhang

Ruiying Zhang

Shengwei Zhang

Zheng Zhang

Zijian Zhao

Yalin Zheng

Jiang Zhu

Jie Zhu

Lei Zhu

Wentao Zhu

Yu Zou

Reyer Zwiggelaar 